\title{
Application of the Wigner-Ville Distribution for the Detection of Rotor Asymmetries and Eccentricity through High-Order
}

\author{
Harmonics \\ V. Climente-Alarcon ${ }^{\mathrm{a} *}$ \\ J. A. Antonino-Daviu ${ }^{\mathrm{a}}$ \\ M. Riera-Guasp ${ }^{\mathrm{a}}$ \\ R. Puche-Panadero ${ }^{a}$ \\ L. Escobar ${ }^{\mathrm{b}}$ \\ a - Polytechnic University of Valencia \\ Instituto de Ingeniería Energética \\ b - Norbert Wiener Center for Harmonic Analysis and \\ Applications \\ P.O.Box 22012, 46071 Valencia, Spain \\ Phone: 0034-96-3877592, Fax: 0034-96-3877599 \\ e-mail: viclial@csa.upv.es \\ * corresponding author
}

\begin{abstract}
The diagnosis of induction machines through the use of methods based on the study of the startup current has become an issue of special interest. These techniques may provide, in certain situations (unbalanced supply voltages, load torque oscillations, variable load...) and for certain faults (broken bars, eccentricity, stator short circuit,) substantial advantages in comparison with the classical method, based on the Fourier spectrum of the steady-state current. Nevertheless, in the case of rotor asymmetries, these transient-based techniques have been mainly focused on the tracing of the lower sideband harmonic (LSH). In this paper, a wideband diagnosis method is proposed, in which the Wigner-Ville distribution is applied to the detection of eccentricity and other high-order components also introduced by the rotor asymmetry. It is shown that the proposed wide band analysis might help to reach a more reliable diagnosis conclusion in cases in which the tracing of commonly used harmonics may be difficult (inter-bar currents, load torque oscillations, non stationary regimes...). An evaluation
\end{abstract}


of the method is carried out through simulations and laboratory tests. The results show the potential of the tool for the detection and quantification of these components as a basis to diagnose such faults.

Keywords: Time-frequency analysis, fault diagnosis, Wigner-Ville distribution, induction motor, condition monitoring, digital filters

\section{INTRODUCTION}

The aim of this paper consists of the development of a method for the application of the Wigner-Ville Distribution (WVD) in order to identify, track and quantify components of interest for predictive maintenance and fault diagnosis of induction motors. Contrary to other procedures developed up to now, this method is particularly focused on the high order components of the startup transient current that reflect rotor asymmetries in an induction machine, as well as the harmonics related to the eccentricity fault that evolve around the fundamental component.

Maintenance of induction motors is an issue of special concern in the industrial environment. Eccentricity under its various modalities (static, dynamic or mixed) is one of the most common faults. Broken rotor bars and, more generally, rotor asymmetries, amount for around $10 \%$ of the failures in such equipment [1]. This fault does not cause an immediate collapse of the machine but it propagates progressively towards the adjacent bars, leading to an irreversible failure, unless condition monitoring is applied.

This is especially problematic in large motors started under high inertias, in which the most favourable conditions for the appearance of this type of faults are present. Moreover, these motors are usually those involving the highest repair costs. 
In the industrial environment, the most widely spread approach for the detection of rotor bar breakages and eccentricities, known as Motor Current Signature Analysis (MCSA) [2]-[4], has some drawbacks, such as the difficulty of being applied to machines under unloaded condition or under unbalanced supply voltages, varying load or load torque oscillations [5]-[7].

Recent methods based on the study of transient processes of the machine and specifically, on the startup transient have been applied to overcome these drawbacks. Due to the time-varying frequency spectrum of the startup current signal, time-frequency decomposition tools have been applied, such as wavelet transforms (WT) [8]-[11], Hilbert-Huang transform (HHT) [12] and other Time Frequency Distributions (TFD), such as Wigner-Ville (WVD) [13]-[15].

Regarding rotor asymmetries, most of these studies have been mainly based on the detection of the lower sideband harmonic (LSH), since it often constitutes the most prominent harmonic introduced by this fault. However, few techniques have focused on the analysis of other high-order components caused by the asymmetry, which constitute the main subject in this work. Indeed, as some authors have claimed [16], the presence of these harmonics in the FFT spectra may help to ratify a diagnosis conclusion in certain situations in which the diagnosis using the main sidebands is not decisive. (This is the case of presence of inter-bar currents, pulsations in the load torque or voltage oscillations.)

The main analysis tool used in this work, the WVD, and other derived time-frequency distributions, have been recently applied for discerning mechanical faults in induction machines thanks to the great amount of information and detail that they are able to provide [15], [17]-[19], especially on high frequency components. However, these studies use modified kernels in order to reduce the artifacts added by this kind of tools [17], [20] and have focused in particular effects of specific faults and online diagnosis. Contrary to that, since those faults do not usually imply an immediate machine failure, the approach followed in this work is aimed to providing a wideband view on the harmonics that 
evolve during transitory operation, especially startups, whilst keeping the computational requirements low. This allows an easier identification of the multiple components of interest, guaranteeing the detection of the fault, and yielding a general sight on the state of the machine in a single look. The proposed procedure uses a filtering stage of constant frequency components prior to the computation of the time-frequency distribution. The reduction of artifacts provided by this pretreatment allows the use of the WVD with no-modified kernel.

For these purposes, the present paper is structured as follows: section 2 introduces the theoretical basis of the Wigner-Ville Distribution which is the signal analysis tool proposed in this paper, section 3 establishes the theoretical evolution during the startup of the components that are involved in the diagnosis approach. Section 4 defines the acquisition and pretreatment procedures of the signal analyzed. In sections 5 and 6 , the validation of the proposed approach is carried out, on simulated and tested signals, respectively, and finally, section 7 summarizes the conclusions of this work.

\section{WIGNER-VILLE DISTRIBUTION}

The Wigner-Ville distribution is a particular case of the Cohen class distributions which yields a time-frequency energy density computed by correlating the signal with a time and frequency translation of itself.

The WVD of a signal $x(t)$ is defined by (1):

$$
W_{X}(t, \omega)=\frac{1}{2 \pi} \int_{-\infty}^{+\infty} x\left(t+\frac{\tau}{2}\right) \cdot x^{*}\left(t-\frac{\tau}{2}\right) \cdot e^{-j \tau \omega} d \tau
$$

where $x^{*}$ denotes the conjugate of $x$. Thus, the Wigner integral is the Fourier transform, with respect to the delay variable $\tau$, of $x(t+\tau / 2) x *(t-\tau / 2)$. This procedure avoids any loss of time-frequency 
resolution, as it happens, for instance, when performing the windowing in the Short Time Fourier Transform [14]. It has Hermitian symmetry in $\tau$ remaining always real [21]

Unlike other recently applied distributions [13], [15], [17], the Wigner-Ville distribution has the desirable property of fulfilling the marginal conditions [21], thus total signal energy can be calculated in time or in frequency using the Plancherel formula:

$$
\|x\|^{2}=\int_{-\infty}^{+\infty}|x(t)|^{2} d t=\frac{1}{2 \pi} \int_{-\infty}^{+\infty}|X(\omega)|^{2} d \omega
$$

The values $\|x(t)\|^{2}$ and $\|X(\omega)\|^{2}$ can be interpreted as energy densities in time and frequency, respectively. This enables the computation of the energy present at a given time-frequency box directly from the WVD output.

Another important characteristic the DWV features is that its first conditional moment for a given time $t_{c}$ mathematically equals the instantaneous frequency [21]:

$$
\frac{d \varphi}{d t}=\langle\omega\rangle_{t_{c}}=\frac{1}{\left|s\left(t_{c}\right)\right|^{2}} \int_{-\infty}^{+\infty} \omega W\left(t_{c}, \omega\right) d \omega
$$

Therefore, it can be computed as the average of all frequencies $\omega$ present in the time-frequency plane at a time $t_{c}$.

In discrete form, the WV distribution is defined by [19]:

$$
W V D(n, k)=\sum_{p=-N}^{N-1} R[n, q] \cdot e^{-\frac{j 2 \pi k q}{N}}
$$

where $R[n, q]$ is the instantaneous correlation given by (5), $n$ is the number of samples of the analytical or interpolated form of the discrete signal $x[n]$ and $q$ is an odd integer.

$$
R[n, q]=x\left[n+\frac{q}{2}\right] \cdot x *\left[n-\frac{q}{2}\right]
$$


Since the instantaneous correlation is centered on a value, the delay $q$ is distributed between the delayed sample $x[n-q / 2]$ and the advanced one, $x[n+q / 2]$; thus being necessary to calculate the values of $x[n]$ at half integer positions using interpolation. In addition, positions at the extremes of $x[n]$ are padded with zeros in order to compute the Fourier transform.

Despite the Wigner-Ville distribution is faster than other exponential algorithms and it has an excellent joint time-frequency resolution [19], its use is hindered by the appearance of outer interferences, when applied to the analysis of multicomponent signals. These interferences, also known as cross terms, appear in the distribution at time instants or frequencies in which there should be no energy.

As a consequence, it is advisable to calculate the WVD on the analytic signal obtained from the Hilbert transform [22] of the real signal, since the Fourier transform of an analytic signal has no negative frequency components and thus, there cannot be any interference between positive and negative frequency ones.

The Hilbert transform of a real signal $x(t)$, such as the phase current, is defined as a convolution with the function $1 / t$, as follows [22]:

$$
H T(x(t))=y(t)=\frac{1}{\pi t} * x(t)=\frac{1}{\pi} \int_{-\infty}^{+\infty} \frac{x(\tau)}{t-\tau} \mathrm{d} \tau
$$

The divergence at $t=\tau$ is allowed for by taking the Cauchy principal value of the integral. By coupling the $x(t)$ and its HT, the so-called analytic signal is created:

$$
\hat{x}(t)=x(t)+j H T(x(t))
$$

The analytic signal has a one-sided Fourier transform, that is, its negative frequencies are null [23]. It retains the positive frequency content of the original signal, while annulating negative frequencies and doubling the DC component. 
In order to further minimize the cross term problem, now appearing between the positive frequency components, authors added in the computation of the Time-Frequency distribution a two dimensional function called kernel, which originates new transforms with different features.

As all these modifications come at the expense of adding some limitations in other desirable properties, the general procedure nowadays consists of selecting the kernel accordingly to the duty to be developed, that is, selecting among all the Cohen class distributions, the best fitted for each task.

Instead of that, this paper proposes an alternative way to minimize the drawback caused by the cross-terms; here, it has been preferred to keep the higher resolution of the WVD -that is, choosing the kernel equal to " 1 " - since the aim of its development is to detect incipient signs of failure. Cross terms are either tolerated or minimized by filtering the signal prior to the analysis, in order to eliminate the constant frequency components and keep only the frequency bands in which the fault effects are present.

\section{HARMONIC FEATURES OF INTEREST IN THE STARTUP CURRENT}

In this section the current components traced during the startup which are relevant for application of the proposed approach are presented:

\subsection{High order harmonics due to a bar breakage}

The two main components often traced for the diagnosis of rotor asymmetries are known as lower sideband harmonic (LSH) and upper sideband harmonic (USH) and their frequencies $f_{s b}$ are given by (8) ( $s=$ slip and $f=$ supply frequency) [2].

$$
f_{s b}=(1 \pm 2 \cdot s) \cdot f
$$

The frequencies of these components are a particular case of the general expressions of the components amplified by the breakage, given by (9) and (10) [24-25]. 


$$
f_{b}=\left(\frac{k}{p}(1-s) \pm s\right) \cdot f \quad \frac{k}{p}=1,3,5 \ldots
$$

From this equation, for each value of $k / p>1$ two components are obtained (high order lower sidebands), whose frequencies at steady state are slightly smaller than the frequency of the current harmonic of $k / p$ order.

Moreover, the pulsations in the speed of the rotor that the above mentioned components cause, trigger further descending harmonics given by (10) [25], which, from multiples of the fundamental frequency, converge towards the main component as the slip is reduced; for $|k|>1$ these harmonics are designated as high order upper sideband harmonics.

$$
f_{b}=(1 \pm 2 \cdot k \cdot s) \cdot f \quad k=1,2,3 \ldots
$$

Apart from these components, other authors [26] have proven the appearance of harmonic components due to the breakage located around the Principal Slot Harmonics (PSH); the frequencies of these PSH components are given by (11) with $N_{r}=$ number of rotor bars; $\lambda=1,2 \ldots ; v=1,3,5 \ldots$ [3]

$$
f_{R S H \pm v}=\left[\frac{\lambda \cdot N_{r}}{p} \cdot(1-s) \pm v\right] \cdot f
$$

During the startup transient, the slip s varies from a value equal to 1 , when the machine is connected, to a value near to 0 (steady-state). Therefore, since the expressions (9) to (11) are function of the slip, the characteristic fault-related frequencies given by these expressions evolve in a particular way for each harmonic. This evolution is linear with the slip thus being possible to characterize them simply by their frequencies when the machine is connected and at steady state.

Tables I and II portray the evolution of the main high frequency lower sideband components amplified by the asymmetry from the connection instant $(s=1)$ to the steady state, assuming ideal unloaded condition ( $\mathrm{s}=0$ ), according to (9). Table I characterizes the components computed using the 
“+" sign in (9), which have positive initial frequencies at startup. In these cases, their frequencies increase from the fundamental frequency value up to their final steady-state value (direct evolution).

Table II presents the lower sideband harmonics computed using the “-” sign in (9). Their absolute frequency value firstly decreases, falling progressively to zero and then increases again until reaching its steady state frequency.

In addition, an easily identifiable descending harmonic is the USH, obtained for $\mathrm{k}=1$ in (10) whose frequency evolves from 150 to near $50 \mathrm{~Hz}$ during the startup.

Finally, Table III shows the main PSH's according to (11) for one of the machines used in section 5 (motor Type I) in the validation of the method. The characteristics of this machine are given in Appendix A.

\subsection{Eccentricity components}

In an induction motor the eccentricity causes a non-uniform air-gap between stator and rotor. Conceptually, two main types of eccentricity exist; the static eccentricity and the dynamic eccentricity.

In the case of the static eccentricity, the position of minimum air-gap remains fixed in space. However, since the dynamic eccentricity appears when the rotation axis of the rotor does not coincide with its geometric axis, the position of minimum air-gap changes with time. In practice both these types of eccentricity usually coexist, which is known as mixed eccentricity.

Several authors have provided expressions for the calculation of the frequencies of the components introduced by static or dynamic eccentricities [27]. If both types of eccentricities are present, low frequency components near the fundamental appear [28]. The frequencies of these components are given by (12), where $f_{r}$ is the frequency of rotation of the rotor.

$$
f_{\text {ecc }}=f \pm k \cdot f_{r} \quad \mathrm{k}=1,2,3 \ldots
$$

An equivalent formula is provided by other authors [1]. 


$$
f_{e c c}=\left[\left(1+m\left(\frac{1-s}{p}\right)\right)\right] . f \quad m= \pm 1, \pm 2, \pm 3 \ldots
$$

Since (13) depends on the slip, as it varies from 1 to almost 0 during the startup, the components associated with the eccentricity evolve in a very characteristic way; the frequency of the first component $(m=-1)$ for a two pole pair machine starts being equal to the supply frequency $f(s=1)$ and decreases along the startup, reaching almost $f / 2$ when the steady-state is reached $(s \approx 0)$. The second component $(m=1)$ has also an initial frequency equal to $f(s=1)$ evolving towards $3 \cdot f / 2(s \approx 0)$ and so on. The tracing of these components during the startup has been proven to be a reliable way for the detection of eccentricities [29], [30]. Table IV summarizes the main components calculated from (12) used in this paper for eccentricity diagnosis.

Once the relevant components of the startup current, included in Tables I-IV, have been characterized, the proposed analysis method will be applied to tested startup currents as a basis for a wideband-based diagnosis of the machine condition, taking advantage of the ability of this tool for the simultaneous time-frequency analysis of high and low frequencies with good frequency resolution.

\section{PRACTICAL APPLICATION OF THE APPROACH}

For validating the proposed approach in the case of a rotor asymmetry, two series of tests were carried out at different winding temperatures using two commercial cage motors (Type I and Type II) whose characteristics are given in Appendix A [31]. On both cases, two rotors, the first being healthy and the second having a rotor bar drilled at its junction with the short circuit ring (Fig. 1), were successively assembled in the same stator. For loading purposes, a DC machine was used (Fig. 2).

A phase current was used as diagnostic signal; this current was acquired using a 15/5, class 0.5 current transformer and a $1 \mathrm{~A}, 60 \mathrm{mV}$ shunt; the resulting voltage signal was captured by means of a 
digital oscilloscope with a sampling frequency $\mathrm{f}_{\mathrm{s}}=5000$ samples/s, and finally transferred to a PC for the analyses.

For the evaluation of the eccentricity, the source of the signal in this case has been a computational model which is based on the magnetic coupled circuits approach [32]. Instead of using the winding function approach, the computation of winding inductances have been carried out following a new technique presented in [33]-[35]. It is based on the computation of the mutual and self inductances of a single conductor, and the superposition of the effects of a phase conductors using circular convolution and the Fast Fourier Transform. This technique greatly reduces the computation time, which is very convenient to perform multiple simulations of a faulty machine under different working conditions.

The signals obtained following the aforementioned procedures underwent two analysis methods; the first one yields an ample time-frequency diagram to provide an insight of the evolution of several fault harmonics, which enhances the possibility of correctly diagnosing the defect; the second one studies a reduced time-frequency box in order to perform a quantification of the energy contained in one of such fault harmonics.

\subsection{Wideband fault detection procedure}

In this case, the processing of the data is divided in three steps:

1) Suppression of the constant components of the tested signal.

First of all, the transient portion of the captured current is filtered in order to remove constant frequency components, related to the fundamental component as well as other stator winding harmonics. This procedure, which eliminates cross terms and yields a clearer representation of the results, is applied gradually, eliminating the main current harmonic in all cases and removing higher order ones as needed. Chebyshev and Elliptic notch filters, as implemented in MATLAB and shown in Appendix B, are suitable for this task. The wide frequency evolution of the fault 
components during the startup overcomes the problems seen in the application of notch filters to the study of rotor asymmetries in stationary operation, that is, the attenuation of the sidebands [36].

When the study is focused on a specific time-frequency box, the signal is also filtered by means of high and low pass Butterworth filters fitted to the limit frequencies. Further, down sampling eases the computational requirements.

2) Computation of the analytic signal

The analytic signal is obtained from the filtered data by means of the Hilbert transform [22], in the second step of the data processing, to avoid the cross terms produced as a result of positive and negative frequency components interfering.

\section{3) Application of the WVD}

Finally, the time range is selected and the WVD of the resulting signal is calculated using the TFTB Toolbox developed by CNRS (France) and Rice University (USA) for MATLAB [37].

\subsection{Quantification procedure}

The quantification procedure proposed in this paper is derived from [39]. It consists of obtaining, following the steps 1 to 3 presented in the section 4.1, a suitable time-frequency box in which a fault component prevails. Using (3), the instantaneous frequency for each time of that time-frequency box is computed. The result is smoothed, firstly discarding the values above and below the frequency limits of the considered time-frequency box and then using a low pass filter. The obtained instantaneous frequency follows the evolution of the prevailing fault harmonic, and hence its energy $e_{f i, b w}$, which reflects the degree of the fault, can be computed by integrating the value of the WVD on a narrow band, whose width is determined empirically, centred for each time in that instantaneous frequency (14): 


$$
e_{f i, b w}=\sum_{t} \sum_{f=f i-b w}^{f i+b w} W V D(f, t)
$$

where $f i$ is the instantaneous frequency for each time $t$ in the time-frequency box considered, $b w$ is the half bandwidth and WVD is the Wigner-Ville distribution computed for that time-frequency box.

The comparison of this value with the energy in the original current waveform for the same time limits yields a quantification parameter:

$$
\gamma_{W, \chi}=10 \cdot \log \left[\frac{2 \sum_{t} i_{j}^{2}}{e_{i f, b w}}\right]
$$

where, $\chi$ is the name of the evaluated component, $i$ is the instantaneous value of the current for the same interval $t$ as in (14), and the doubling of the energy when the analytical signal is computed is considered.

Other quantification techniques, such as the one proposed in [24] based in the discrete Wavelet transform, evaluate the energy present in a complete time-frequency box. However, in the proposed procedure the band where the energy is integrated is centred on the fault component, thus minimizing the contributions of other small amplitude fault harmonics, always present in the startup current due to manufacturing tolerances, and therefore yielding a more stable fault indicator.

\section{SIMULATION RESULTS}

As introduced in Table IV, for a mixed eccentricity fault, harmonics around the main component appear. These harmonics evolve following a characteristic pattern moving away from this component as slip decreases [24].

Fig. $3 \mathrm{a}$ and $3 \mathrm{~b}$ show results of the application of the proposed fault detection approach to a simulated startup transient of a four pole machine in the cases of healthy machine and machine with mixed 
eccentricity (30\% static eccentricity, $30 \%$ dynamic eccentricity). The simulated currents undergo the treatment stages shown in section 4.1: the main and other constant frequency harmonics are removed by filtering, and in this case, the cross terms are further minimized restricting the area of interest to frequencies below $300 \mathrm{~Hz}$, before obtaining the analytical signal and computing its WVD.

As Fig. 3b shows, the proposed method is able to identify the characteristic evolution of the components EC 25 (a1), EC 75 (a2), EC 100 (a3) and EC 125 (a4) caused by this fault.

In addition, the proposed quantification approach shown in section 4.2 , is applied to the time frequency box defined by the yellow dotted line in Fig. 3b. Since the evolution of the EC 75, EC 100 and EC 125 harmonics is symmetrical in each time and the amplitude of the EC 100 is greater, the instantaneous frequency engages this last one, yielding the computation of the quantification parameter $\gamma_{\mathrm{W}, \mathrm{EC}} 100$ a value of $30.1 \mathrm{~dB}$.

\section{EXPERIMENTAL RESULTS}

For the validation of the proposed approach in the detection and quantification of a bar breakage, experimental tests on two commercial motors, whose characteristics are shown in Appendix A, have being carried out. Depending on the time-frequency box selected, and the depth of the filtering stage, the study is focused on specifically evolving harmonics.

\subsection{Identification of the PSH components}

One of the questions that arises when intending to study the high order harmonics in an induction motor is the necessity of identifying the PSH components, which are significant in this part of the spectrum, either with the aim of discarding them as fault components or in order to detect a defect by studying their associated fault related sidebands. 
Fig. 4 shows the stator current measured in a startup of the tested machine in healthy condition. Fig. 5 shows its WVD. In this case, a light filtering stage has been applied, removing only the main current harmonic, prior to obtaining the analytic signal and the time-frequency distribution. The trace of the PSH+1 harmonic (b2), between PSH-1 (b3) and PSH+3 (b1), is easily discernible simply taking into account the frequencies corresponding to them at the connection instant and at stationary operation, given in Table III. Furthermore, the number of rotor bars can be inferred then from (11); this information may be useful for industrial maintenance.

\subsection{Detection and quantification of the high-order sideband harmonics}

High order sideband harmonics can be subsequently detected in the startup transient, when the frequency of the PSH increases over them. Therefore, to reduce computational needs and provide a better representation, the WVD is applied to the time interval of the startup waveform between 1000 and 2200 ms, which is shown in Fig. 6.

The data pretreatment in this case has also been wider. The constant frequency components at frequencies of 50, 150, 250 and $350 \mathrm{~Hz}$ are practically eliminated by means of Chebyshev and Elliptic stop band filters. This procedure, as commented before, minimizes the appearance of artifacts in the time-frequency box of study.

Additionally, the signal is centered, low pass filtered and down sampled by a factor of 4 .

Fig. 6 shows that the main Rotor Slot Harmonics have decreased in amplitude and/or reached the higher frequencies above the limit of the box, although the trace of the PSH-1 (c1) can still be observed. The rests of the main current harmonic (c2) and the $250 \mathrm{~Hz}$ winding harmonic (c3) can also be identified.

Fig. 7 has been obtained under identical conditions as those of Fig. 6 but using the startup current of the machine with one broken bar. In this case, the LSH associated with the main $50 \mathrm{~Hz}$ 
harmonic, LSH 50 (d1), prevails during the second part of the transient stage, as illustrated in Fig. 7. Two LSH related to the $150 \mathrm{~Hz}$ are also perceptible, even using a linear scale to represent the results (d2).

In addition, the LSH linked to the main current harmonic (d1) can be damped using a high pass filter, yielding even a better vision of the LSH and USH associated to high-order harmonics as it is shown in Fig. 8, in which the graphic has been enhanced using a logarithmic scale for the amplitude representation.

In this case, the LSH related to the $250 \mathrm{~Hz}$ current harmonic (e1) predicted by (9) and the USH+S 150 (e3) predicted by (10) are clearly shown; the LSH related to the $150 \mathrm{~Hz}$ current harmonic (e2) predicted by (9) is also discernible.

Furthermore, applying the proposed quantification procedure to a time-frequency box whose limits are determined by the yellow dotted line in Fig 8, the energy contained in the LSH 250 harmonic is evaluated, yielding values for $\gamma_{\mathrm{W}, \mathrm{LSH}} 250$ of $60.7 \mathrm{~dB}$ for a healthy machine and 42.2 $\mathrm{dB}$ for a motor having a broken bar.

The proposed WVD methodology can be used to study any transient regime which involves speed variations; for instance, in the case of a plug stopping, the slip varies from practically 2 (at the moment of the phases inversion) to 1 (when the rotor stops). Thus, the lower sideband harmonic related to the main supply frequency (LSH 50) changes from near $150 \mathrm{~Hz}$ to $50 \mathrm{~Hz}$ (10) during this transient. Similarly, the PSH's reduce their frequency (11) reaching the PSH+1 also the frequency of the fundamental component when the machine comes to a standstill.

Fig 9a shows the tested stator current during a transient involving a plug stopping followed by a startup in a four pole healthy machine; Fig. 9b shows the WVD of the current of Fig.9a The first 500 milliseconds after the connection have been omitted in order to avoid the 
electromagnetic transient. PSH's evolution leads to a characteristic "V" shape as the rotor decelerates and accelerates again, with PSH-1 (f1) prevailing over the rest of the rotor slot harmonics.

Similarly, Fig. 9c presents the analysis of the same transient for the induction motor, now having a broken bar. In this case, in addition to the PSH, the LSH 50 (f2) is clearly visible progressing from $150 \mathrm{~Hz}$ to $50 \mathrm{~Hz}$ as the rotor stops, then continuing reducing its frequency again up to $0 \mathrm{~Hz}$ to finally evolve back to its steady state value, almost $50 \mathrm{~Hz}$.

The proposed approach was also tested on a different type of motor (motor Type II) with one pole pair and 20 rotor bars. The characteristics of this motor are given in Appendix A. The combined filtering and WVD procedure has been able to detect bar damage in this motor.

Fig. 10a shows no fault related high order harmonics during the startup of this machine in healthy condition; only the rotor slot components PSH-1 (g1), PSH+1 (g2), PSH+3 (g3) are present.

However, when a rotor having a broken bar is tested, the energy in this time-frequency-box changes (Fig. 10b) and the trace of a LSH 250 (g4) clearly appears. Two descending harmonics (g5-g6) can also be observed.

As in the case of the two pole pair motor, the pretreatment of the sampled current obtained during the startup of the one pole pair induction machine has been wider, being removed frequencies below $50 \mathrm{~Hz}$ by a Butterworth high band pass filter before obtaining the analytical signal and the WVD.

In addition, the quantification procedure is applied to the time-frequency box whose limits are defined by the yellow dotted line in Fig 10. In this case, the method operates in nearly optimal conditions tracking and evaluating the energy of the ascending LSH 250, and yielding a value of 
$51.6 \mathrm{~dB}$ for a healthy machine, since this harmonic is present in the startup of a non-faulty machine due to manufacturing tolerances, and $33.3 \mathrm{~dB}$ for a machine suffering a broken bar. Fig. 11 a) shows the detected instantaneous frequency, and Fig. 11 b) the energy density computed in a band centered on its trace and having a bandwidth of $8 \mathrm{~Hz}$.

\section{CONCLUSIONS}

This paper introduces a new methodology for the diagnosis of induction motors fed at constant frequency, based on a wideband analysis of components within the stator current under variable speed transient conditions via the Wigner Ville Distribution, especially focused in rotor asymmetries and eccentricities.

The proposed procedure provides a wideband detection technique for obtaining a general sight of the evolution of the fault related stator current components under transient conditions, which allows reliable fault detection. In addition, a quantification method able to compute during transient operation the energy contained in a specific fault harmonic, is presented. This technique minimizes the contribution of other components sharing the same frequency band.

In comparison with other time-frequency techniques, the WVD provides a continuous plot, overcoming the vision in bands associated with the discrete Wavelet procedures; a better resolution and preserves the marginal conditions. This enables the precise tracking of varying frequency fault components through much of their evolution, its identification, and the evaluation of their energy by computing the points of interest.

Unlike other TFD-based methods, the proposed approach does not use kernels to minimize the artifacts in the resultant time-frequency plots. A method based on filtering of non significant components of the current is proposed and tested. It is developed in two steps. Firstly, a series of stop- 
band filters are applied to the signal for suppressing constant frequency components (as the fundamental component and winding harmonics) not related to the fault. Second, the resulting signal is filtered again using both a high-pass and a low-pass filter, in such a way that only the frequency band of the signal in which the fault related components are predominant, is considered. Finally the WVD is applied to the resulting signal. Suitable time-frequency boxes obtained by this procedure allow the tracking of the frequency varying fault components by the evaluation of the instantaneous frequency and the integration of energy in a band centered in its path.

The tests carried out on two different types of commercial motors prove that the proposed methodology successfully minimizes the effect of artifacts and enables a clear identification and quantification of the fault related components in the case of machine with broken bars.

In addition, a preliminary evaluation of the suitability of the method for the detection of mixed eccentricity from the analysis of the startup current has been carried out by using a simulated eccentric machine. The approach has been proven to be able to detect the characteristic low and high order components caused by this fault.

\section{APPENDIX A}

Motor type I: Star connected, rated voltage (Un): 400V, rated power (Pn): $1.1 \mathrm{~kW}, 2$ pole pairs, Stator rated current (I1n): 2.7A, rated speed (nn): 1410 rpm. Number of rotor bars: 28.

Motor type II: Star connected, rated voltage (Un): 400V, rated power (Pn): $1.5 \mathrm{~kW}, 1$ pole pair, Stator rated current (I1n): 3.25 A rated speed (nn): 2860 rpm. Number of rotor bars: 20. Total inertia constant of the group: $0.11 \mathrm{~kg}-\mathrm{m} 2$.

\section{APPENDIX B}


For instance, to remove the main current component, the MATLAB code that implements a suitable stop band filter is:

$[\mathrm{B}, \mathrm{A}]=\operatorname{cheby} 1\left(2,0.5,[0.0170 .023],{ }^{\prime}\right.$ stop' $)$

wave=filter(B,A,wave);

Being 2 the half-order of the filter, 0.5 the peak-to-peak ripple in decibels in the pass band, and 0.017-0.023 the lower and upper limits of the frequency to be removed, taking into account that 1 is the top frequency, that is, half the sampling rate.

\section{REFERENCES}

[1] W.T. Thomson, M. Fenger, "Current signature analysis to detect induction motor faults" IEEE Ind. Appl. Magazine, pp. 26-34, July/August 2001.

[2] G.B Kliman, J Stein, and R.D. Endicott, "Noninvasive Detection of Broken Rotor Bars in Operating Induction Motors,” IEEE Transactions on Energy Conversion, vol. 3, no. 4, December 1988, pp. $873-879$

[3] R Cameron et W.T. Thomson and A.B. Dow, "Vibration and current monitoring for detecting airgap eccentricity in large induction motors," IEE Proceedings, vol. 133, pt. B, no. 3, May 1986, pp. 155-163.

[4] D. G. Dorrell, W. T. Thomson, and S. Roach, "Analysis of airgap flux, current, vibration signals as a function of the combination of static and dynamic airgap eccentricity in 3-phase induction motors,” IEEE Trans. Ind. Appl., Vol. 33, No. 1, Jan./Feb. 1997, pp. 24-34

[5] V. Climente-Alarcon, J. A. Antonino-Daviu, M. Riera-Guasp, J. Pons-Llinares, J. Roger-Folch, P. Jover-Rodriguez, A. Arkkio, "Transient tracking of low and high-order eccentricity related components in induction motors via TFD tools," Mechanical Systems and Signal Processing, no. 25, February 2011, pp. 667-679 
[6] J. Cusidó, L. Romeral, J.A. Ortega, A. Garcia, J.R. Riba, "Wavelet and PDD as fault detection techniques", Electric Power Systems Research, Vol. 80, no.8, pp. 915-924, August 2010

[7] S. Nandi, S. Ahmed, H. A. Toliyat, "Detection of Rotor Slot and Other Eccentricity Related Harmonics in a Three Phase Induction Motor with Different Rotor Cages”, IEEE Transactions on Energy Conversion, vol. 16, no. 3, September 2001.

[8] S.H. Kia, H. Henao and G.A. Capolino, "Diagnosis of Broken Bar Fault in Induction Machines Using Discrete Wavelet Transform without Slip Estimation”, Conference Record of the 2007 IEEE Industry Applications Conference, 2007. 42nd IAS Annual Meeting. 23-27 Sept. 2007 Page(s):1917 $-1922$.

[9] J. Pons-Llinares, J. A. Antonino-Daviu, M. Riera-Guasp, M. Pineda-Sanchez, and V. ClimenteAlarcon, "Induction motor diagnosis via frequency B-splines," IEEE Transactions in Industrial Electronics, vol. 58, no. 5, May 2011, pp. 1530-1544

[10] A. Ordaz-Moreno, R. Romero-Troncoso, J.A. Vite-Frías, J. Riviera-Gillen, A.García-Pérez, "Automatic online diagnostic algorithm for broken-bar detection on induction motors based on Discrete Wavelet Transform for FPGA implementation", IEEE Transactions on Industrial Electronics, Vol. 55, no.5, May 2008, pp.2193-2200

[11] M. Riera-Guasp, J.A. Antonino-Daviu, J. Rusek, J. Roger-Folch, "Diagnosis of rotor asymmetries in induction motors based on the transient extraction of fault components using filtering techniques", Electric Power Systems Research, Vol. 79, no. 8, August 2009, pp 1181-1191

[12] J.A. Antonino-Daviu, M. Riera-Guasp, M. Pineda-Sanchez and R.B. Perez, "A critical comparison between DWT and Hilbert-Huang-based methods for the diagnosis of rotor bar failures in induction machines" IEEE Transactions on Industry Applications, Vol.45, no. 5, September/October 2009, pp. 1794-1803 
[13] S. Rajagopalan, J.A. Restrepo, J.M. Aller, T.G. Habetler, and R.G. Harley, "Wigner-Ville distributions for detection of rotor faults in brushless DC (BLDC) motors operating under nonstationary conditions". IEEE International Symposium on Diagnostics for Electric Machines, Power Electronics and Drives, 2005. SDEMPED 2005.

[14] J. Rosero, J.A.O. Romeral, L. Romeral, E. Rosero, "Short circuit fault detection in PMSM by means of empirical mode decomposition (EMD) and wigner ville distribution (WVD)" Applied Power Electronics Conference and Exposition, 2008. APEC 2008. Twenty-Third Annual IEEE.

[15] M. Blodt, D. Bonacci, J. Regnier, M. Chabert, J. Faucher, "On-Line Monitoring of Mechanical Faults in Variable-Speed Induction Motor Drives Using the Wigner Distribution," IEEE Transactions on Industrial Electronics, Vol. 55, No. 2, February 2008.

[16] M. Fernández Cabanas, M. García Melero, G. Alonso Orcajo, J. M. Cano Rodríguez, J. Solares Sariego, in: Marcombo-Boixareu (Ed.), Maintenance and Diagnosis Techniques for Rotating Electric Machinery, ABB Service, S.A., Barcelona, 1999.

[17] M. Blödt, J. Regnier, and J. Faucher, "Distinguishing load torque oscillations and eccentricity faults in induction motors using stator current wigner distributions" IEEE Transactions on Industry Vol. 45, no. 6, September 2009, pp. 1991-2000

[18] B. Trajin, J. Regnier, M. Chabert, J. Faucher, "Wigner Distribution for the Diagnosis of High Frequency Amplitude and Phase Modulations on Stator Currents of Induction Machine.” IEEE International Symposium on Diagnostics for Electric Machines, Power Electronics and Drives, 2009. SDEMPED 2009.

[19] J.A Rosero, L. Romeral, J. A. Ortega, E. Rosero, "Short-Circuit Detection by Means of Empirical Mode Decomposition and Wigner-Ville Distribution for PMSM Running Under Dynamic Condition" IEEE Transactions On Industrial Electronics, Vol. 56, No. 11, November 2009 
[20] S. Rajagopalan, J.A. Restrepo, J.M. Aller, T.G. Habetler, and R.G. Harley, "Nonstationary

Motor Fault Detection Using Recent Quadratic Time-Frequency Representations". IEEE Transactions on Industry Applications, Vol. 44, pp. 735-744, 2008.

[21] L. Cohen, Time-Frequency Analysis. A.V. Oppenheim, Ed. Prentice Hall Signal Processing Series, New Jersey, 1995.

[22] M. Pineda-Sanchez, M. Riera-Guasp, J.A. Antonino-Daviu, J. Roger-Folch, J. Perez-Cruz, R. Puche-Panadero, "Instantaneous Frequency of the Left Sideband Harmonic During the Start-Up Transient: A New Method for Diagnosis of Broken Bars" IEEE Transactions on Industrial Electronics, Vol. 56, no.11, November 2009, pp.4557-4570

[23] N. Wonchul and P. J. Loughlin, "When is instantaneous frequency the average frequency at each time?," IEEE Signal Processing Letters, vol. 6, 1999, pp. 78-80.

[24] M. Riera-Guasp, J.A. Antonino-Daviu, M. Pineda-Sanchez, R. Puche-Panadero, J. Perez-Cruz, "A general approach for the transient detection of slip-dependent fault components based on the discrete wavelet transform", IEEE Transactions on Industrial Electronics, Vol. 55, no. 12, December. 2008, pp. 4167-4180

[25] A. Bellini, F. Filippetti, G. Franceschini, C. Tassoni, G.B. Kliman, "Quantitative Evaluation of Induction Motor Broken Bars by Means of Electrical Signature Analysis“, IEEE Transactions on Industry Applications, Vol. 37, no. 5, September/October 2001, pp 1248-1255

[26] A. Khezzar, M.H. Kaikaa, M. K. Oumaamar, M. Boucherna, H.Razik, "On the use of slot harmonics as a potential indicator of rotor bar breakage in the induction machine", IEEE Transactions on Industrial Electronics, Vol. 56, No. 11, November 2009, pp 4592-4605.

[27] S. Nandi, H.A. Toliyat, "Condition monitoring and fault diagnosis of electrical motors- A review”, IEEE Transactions on Energy Conversion, Vol. 20, No. 4, December 2005, pp.719-729. 
[28] J. Faiz, B. M. Ebrahimi, B. Akin, H. A. Toliyat, "Dynamic analysis of mixed eccentricity signatures at various operating points and scrutiny of related indices for induction motors," Electric Power Applications, IET, vol. 4, no. 1, 2010, pp. 1 - 16

[29] J. Antonino-Daviu, P. Jover, M. Riera-Guasp, J. Roger-Folch and A. Arkkio, "DWT Analysis of Numerical and Experimental Data for the Diagnosis of Dynamic Eccentricities in Induction Motors", Mechanical Systems and Signal Processing, Elsevier, Vol. 21, No. 6, August 2007, pp. $2575-2589$

[30] J. Antonino-Daviu, P. Jover Rodriguez, M. Riera-Guasp, M. Pineda-Sánchez,A. Arkkio, "Detection of Combined Faults in Induction Machines with Stator Parallel Branches through the DWT of the startup current" Mechanical Systems and Signal Processing, Elsevier, Vol. 23, No. 7, October 2009, pp 2336-2351

[31] Climente-Alarcon, V.; Antonino-Daviu, J.A.; Riera, M.; Puche-Panadero, R.; Escobar, L.A., "Wigner-Ville Distribution for the Detection of High Order Harmonics due to Rotor Asymmetries." IEEE International Symposium on Diagnostics for Electric Machines, Power Electronics and Drives, 2009. SDEMPED 2009

[32] J. Faiz, I. T. Ardekanei, and H. A. Toliyat, "An evaluation of inductances of a squirrel-cage induction motor under mixed eccentric conditions," IEEE Transactions Energy Conversion, vol. 18, pp. 252-258, 2003.

[33] J. R. Folch, J. Pérez Cruz, M. Pineda Sanchez, and R. Puche Panadero, "Very fast and easy to compute analytical model of the magnetic field in induction machines with distributed windings," Advanced Computer Techniques in Applied Electromagnetics. vol. 30, pp. 72-79, 2008.

[34] J. Roger-Folch, M. Pineda-Sanchez, J. Perez-Cruz, M. Riera-Guasp, R. Puche-Panadero, and J. A. Antonino-Daviu, "Calculation of winding inductances via magnetic potential vector, discrete 
circular convolution and fast fourier transform," 14th International Symposium on Electromagnetic Fields ISEF 09, Arras, France, 2009, pp. 33-36.

[35] M. Pineda-Sanchez, V. Climente-Alarcon, R. Riera-Guasp, R. Puche-Panadero, and J. PonsLlinares, "Enhanced Simulink Induction Motor Model for Education and Maintenance Training," in The 2nd International Conference on Education, Training and Informatics, ICETI 2011, Orlando, FL, 2011.

[36] B. Ayhan, H. J. Trusell, M-Y, Chow, and M-H. Song, "On the Use of a Lower Sampling Rate for Broken Rotor Bar Detection With DTFT and AR-Based Spectrum Methods," IEEE Transactions on Industrial Electronics, vol. 55, no. 3, March 2008, pp. 1421-1434.

[37] F. Auger, P. Flandirn, P. Gonçalvès, O. Lemoine, “Time Frequency Toolbox to Use with Matlab” CNRS (France) \& Rice University (USA) 1995-1996 http://tftb.nongnu.org/

[38] M. Pineda-Sanchez, M. Riera-Guasp, J. Pons-Llinares, V. Climente-Alarcon y J. Perez-Cruz, Diagnosis of induction machines under transient conditions through the Instantaneous Frequency of the fault components, XIX International Conference on Electrical Machines (ICEM), Rome, 2010.

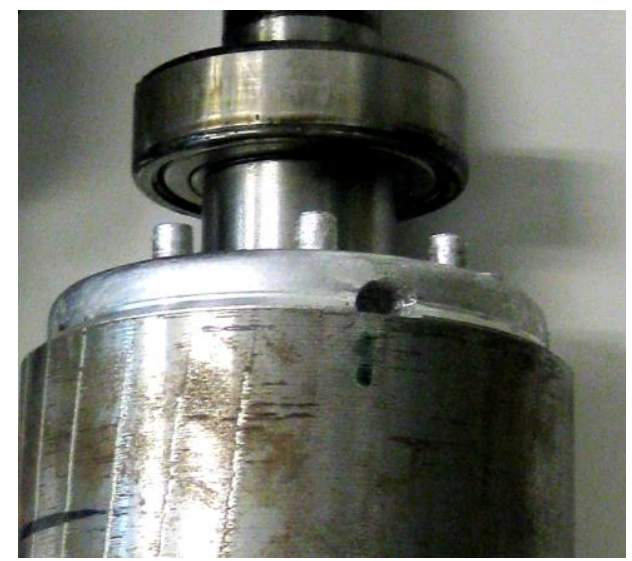

Fig. 1. Rotor bar drilled at one of its ends. 


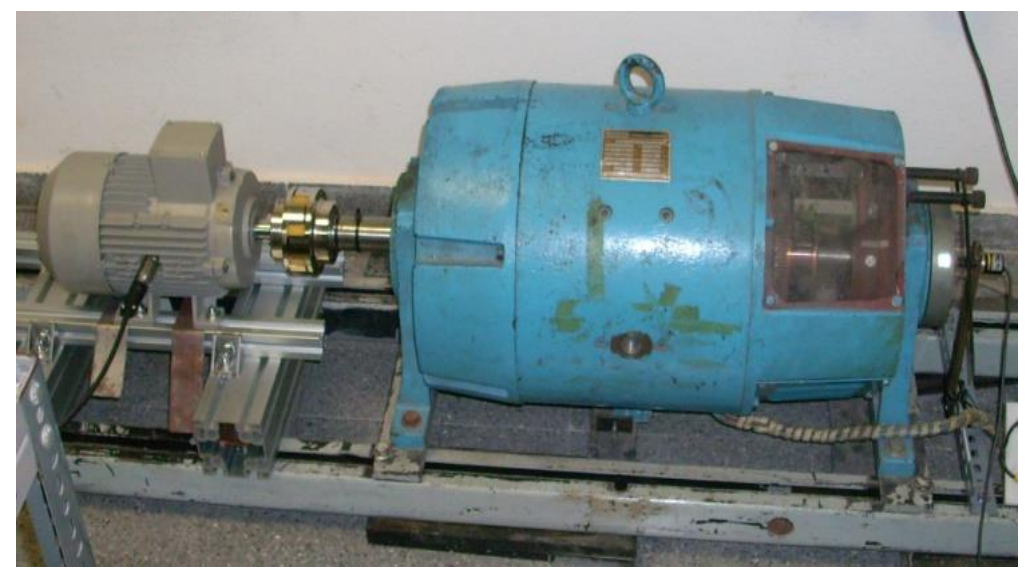

Fig. 2. Experimental test stand.

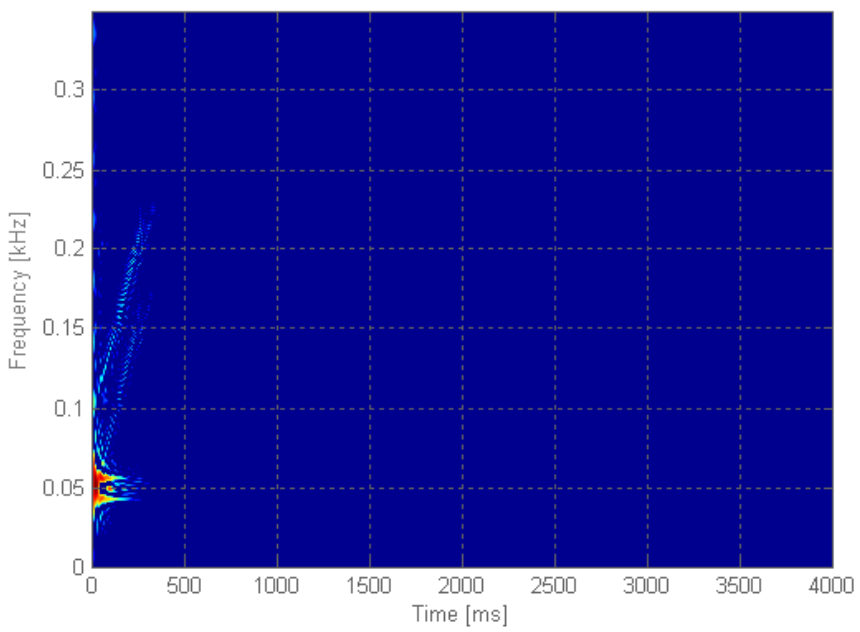

a)

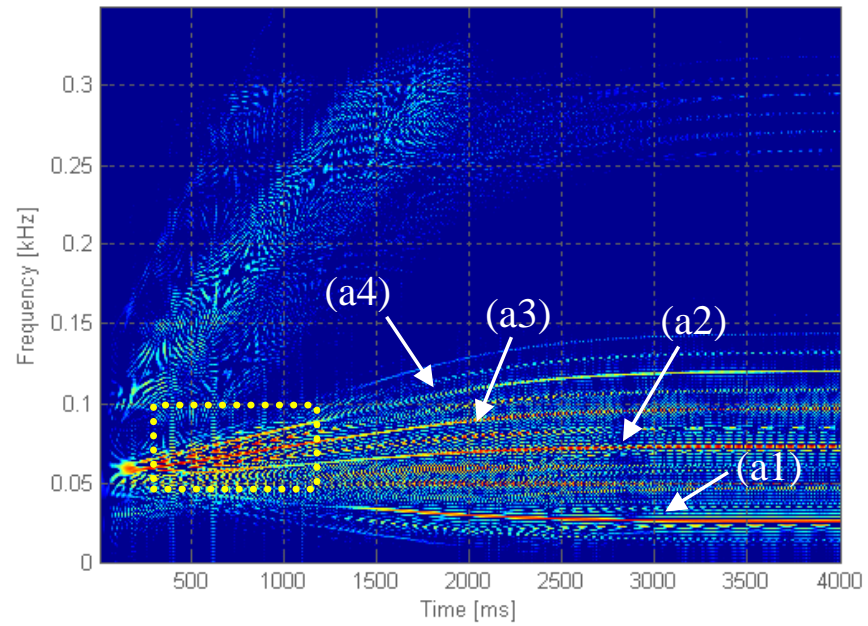

b)

Fig. 3. Wigner-Ville distribution for a simulation of a healthy four pole motor a) and a simulation of the same motor with eccentricity b), logarithmic scale. 


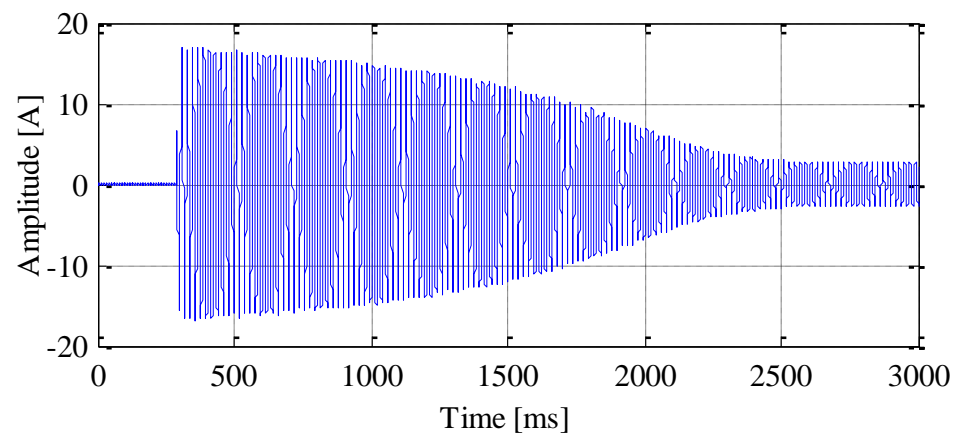

Fig. 4. Startup current waveform of a healthy four pole motor.

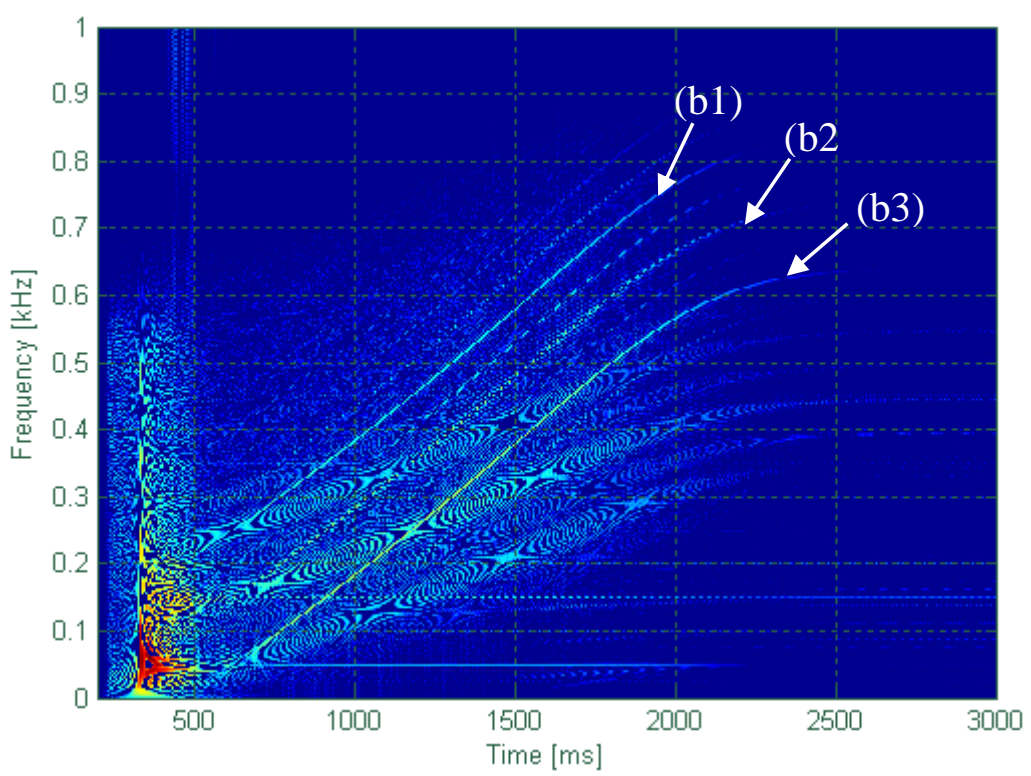

Fig. 5. Wigner-Ville distribution for a four pole motor with a twenty-eight bar rotor, for the startup shown in Fig 4, logarithmic scale. 


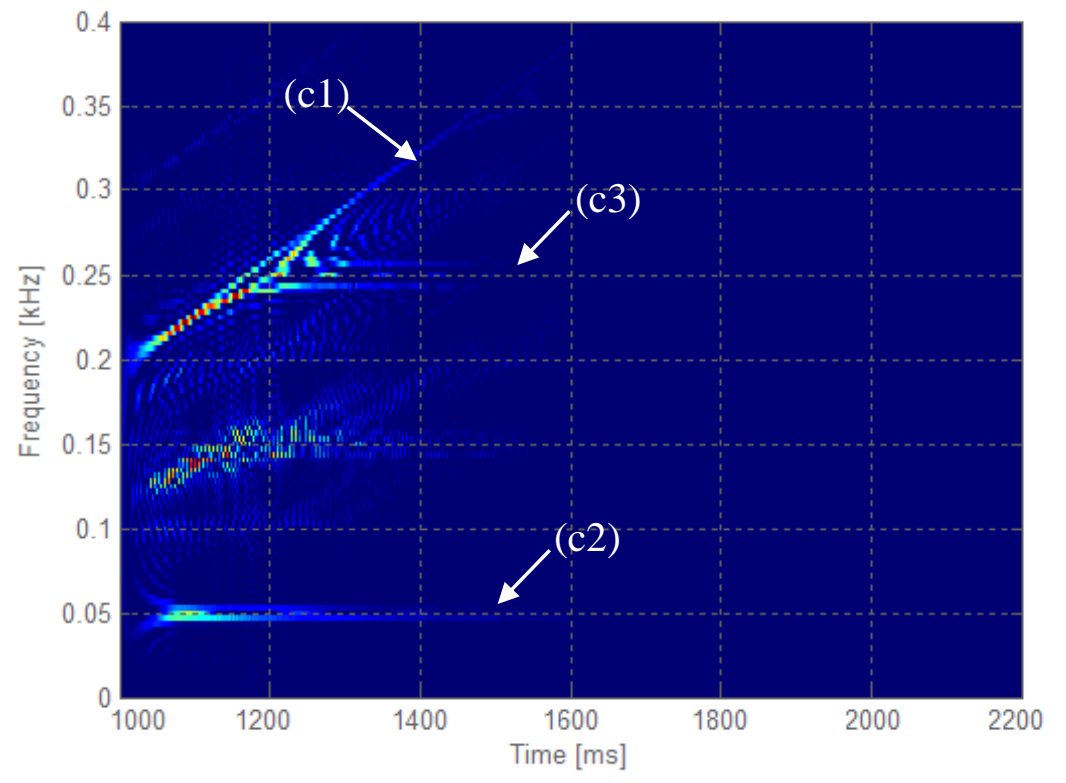

Fig. 6. WVD for a healthy four pole motor startup, linear scale.

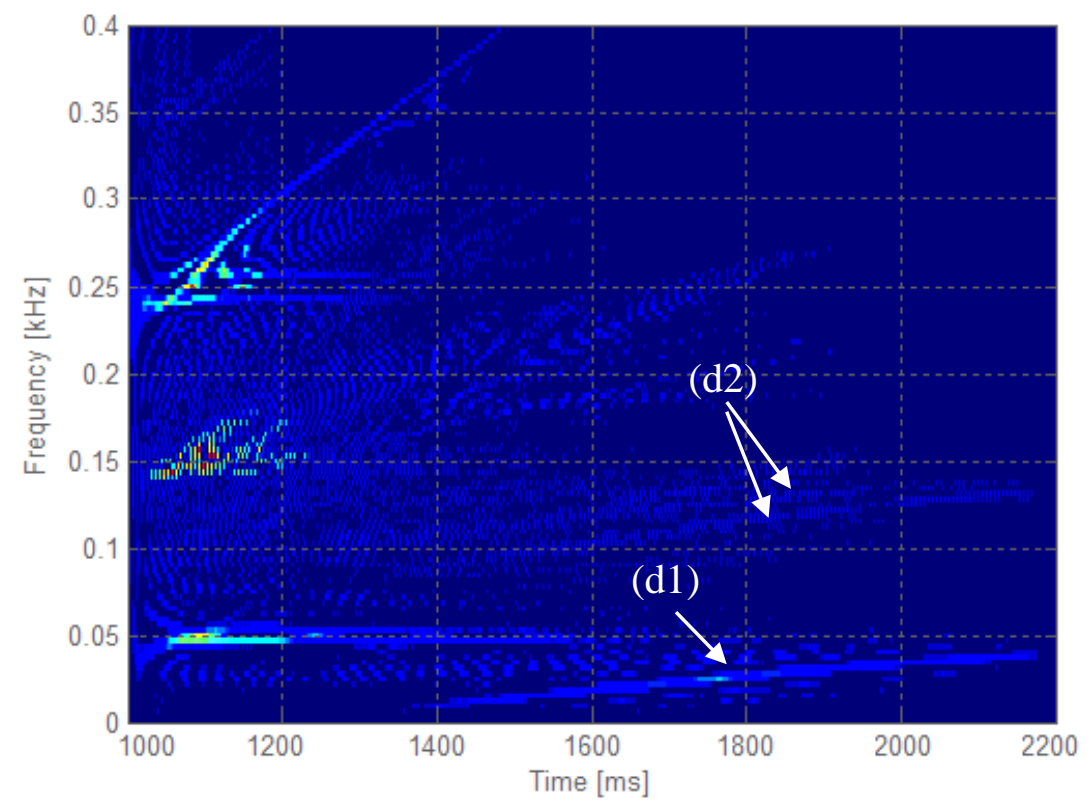

Fig. 7. WVD of the startup transient for a four pole motor having a broken bar, linear scale. 


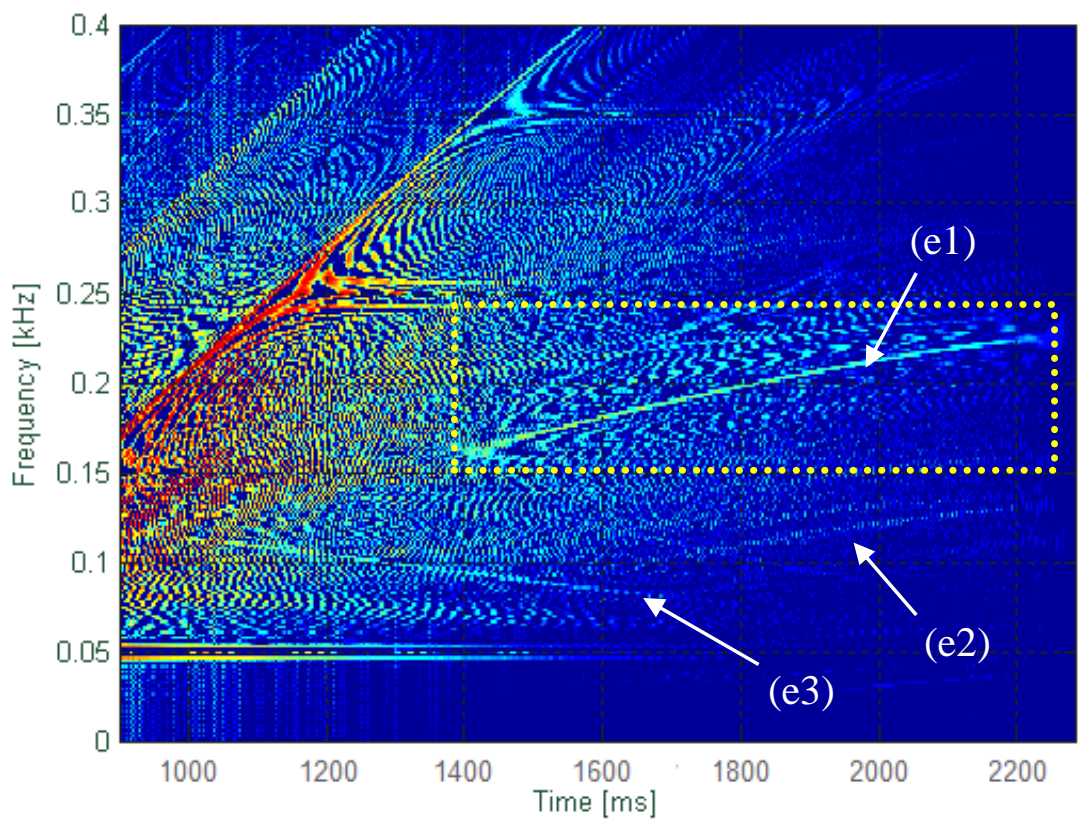

Fig. 8. Wigner-Ville distribution for a four pole motor having a broken bar, logarithmic scale.

Low frequencies have been removed.

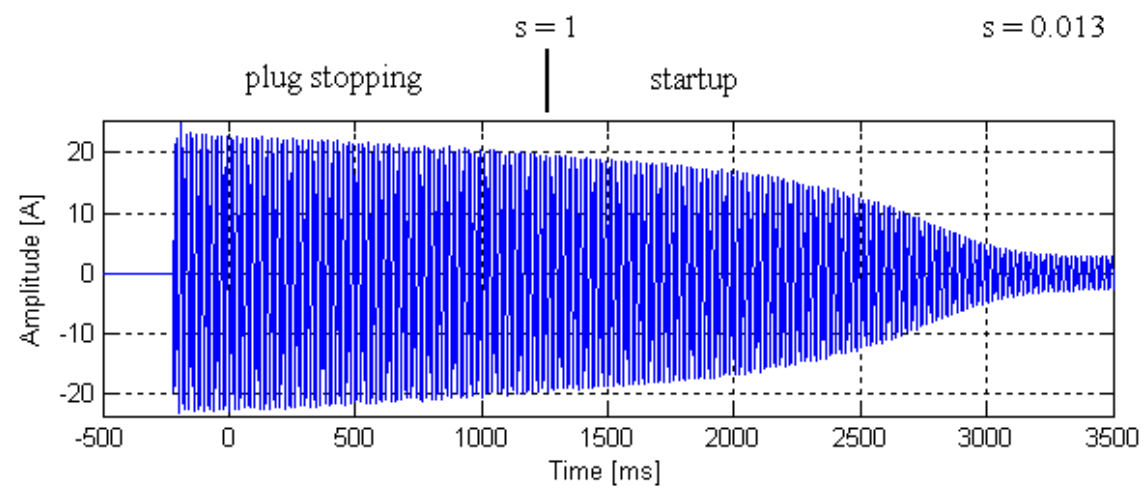

a) 


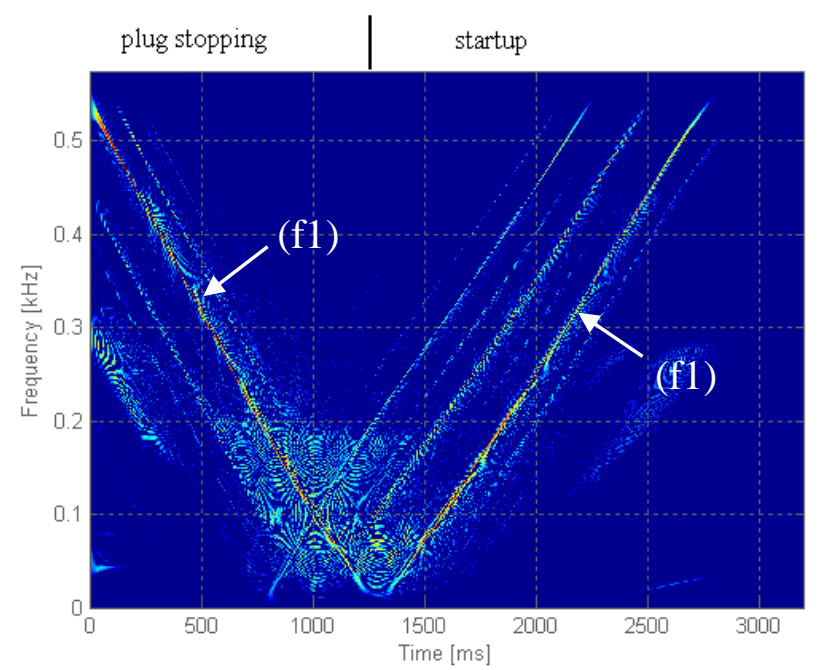

b)

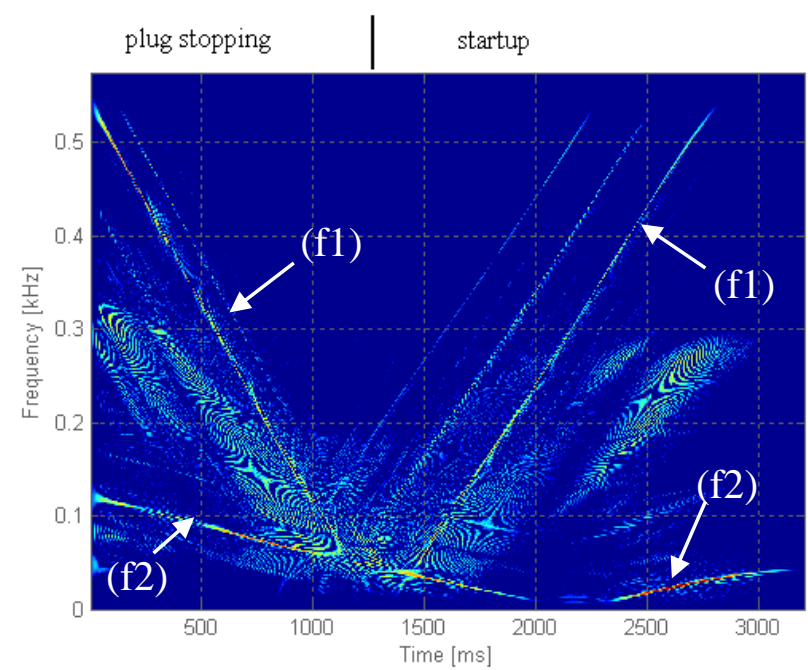

c)

Fig. 9. Waveform a) and Wigner-Ville distribution for a healthy four pole motor b), and WVD of a faulty one having a broken bar c) during plug stopping and startup, logarithmic scale.

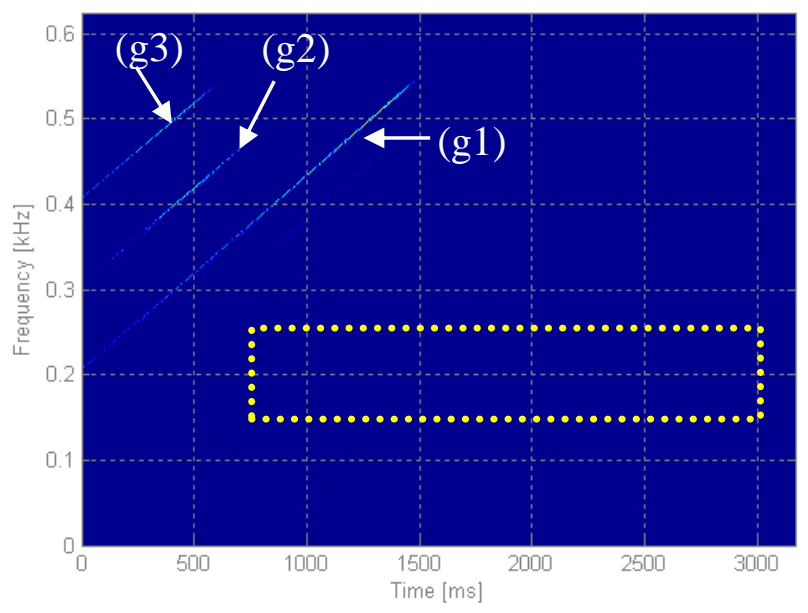

a)

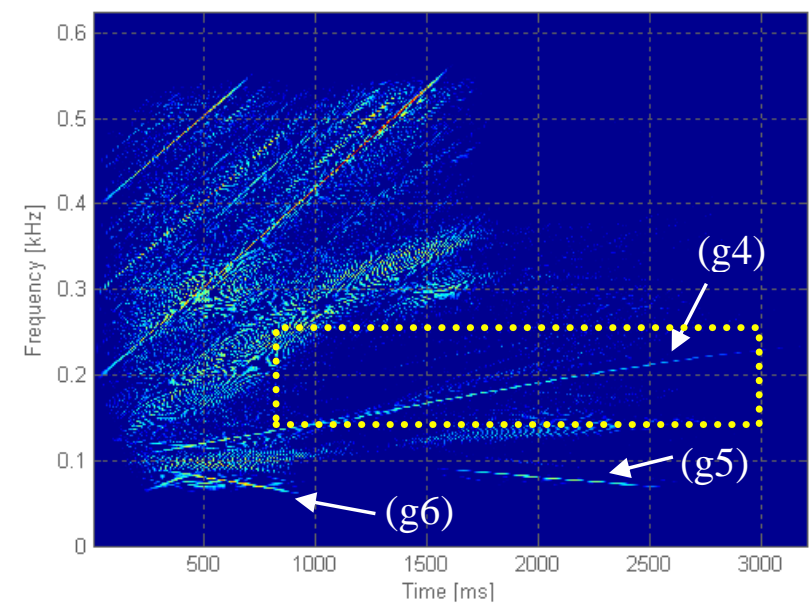

b)

Fig. 10. Wigner-Ville distribution for a healthy two pole motor startup, logarithmic scale a) and a motor having a broken bar b), logarithmic scale. 


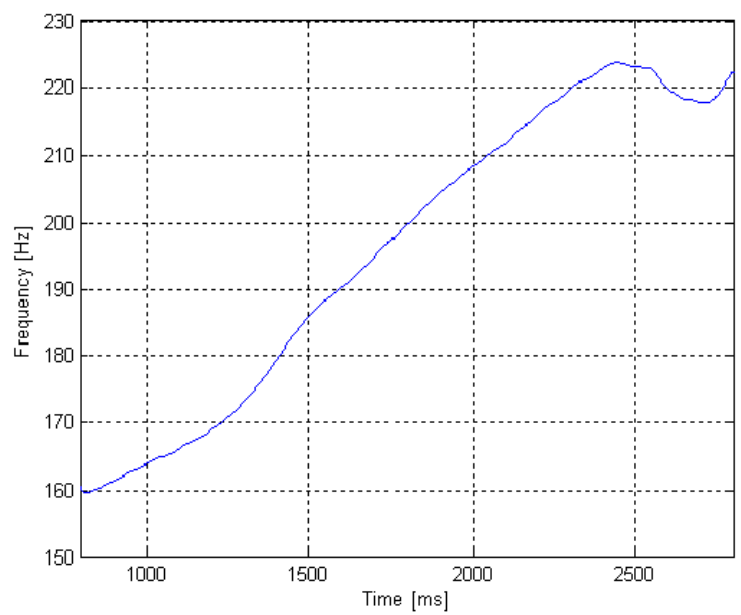

a)

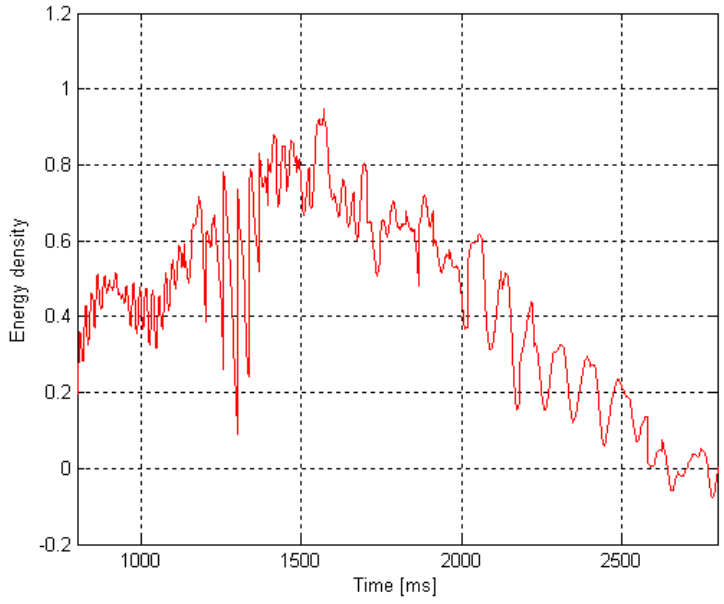

b)

Fig. 11. Quantification of the energy contained in the LSH 250 shown in the yellow dotted box of Fig $10 \mathrm{~b}$ ). Smoothed instantaneous frequency, a), and the computed energy density in a $8 \mathrm{~Hz}$ width band centered on its trace, $b)$. 
Table I. High frequency lower sidebands amplified by the asymmetry according to (9) (direct evolution).

\begin{tabular}{|c|c|c|c|c|}
\hline & $\boldsymbol{k} / \boldsymbol{p}$ & $f \cdot\left(\frac{k}{p} \cdot(1-s)+s\right)$ & $\begin{array}{c}f_{b}(s=1) \\
\mathrm{Hz}\end{array}$ & $\begin{array}{c}f_{b}(s=0) \\
\mathrm{Hz}\end{array}$ \\
\hline $\mathrm{LSH}+\mathrm{S} 150$ & 3 & $f \cdot(3-2 \cdot s)$ & 50 & 150 \\
\hline $\mathrm{LSH}+\mathrm{S} \mathrm{250}$ & 5 & $f \cdot(5-4 \cdot s)$ & 50 & 250 \\
\hline $\mathrm{LSH}+\mathrm{S} \mathrm{350}$ & 7 & $f \cdot(7-6 \cdot s)$ & 50 & 350 \\
\hline
\end{tabular}

Table II. Lower sidebands amplified by the asymmetry according to (9) (indirect evolution).

\begin{tabular}{|c|c|c|c|c|}
\hline & $\boldsymbol{k} / \boldsymbol{p}$ & $f \cdot\left(\frac{k}{p} \cdot(1-s)-s\right)$ & $\begin{array}{c}f_{b}(s=1) \\
\mathrm{Hz}\end{array}$ & $\begin{array}{c}f_{b}(s=0) \\
\mathrm{Hz}\end{array}$ \\
\hline LSH & 1 & $f \cdot(1-2 \cdot s)$ & -50 & 50 \\
\hline LSH-S 150 & 3 & $f \cdot(3-4 \cdot s)$ & -50 & 150 \\
\hline LSH-S 250 & 5 & $f \cdot(5-6 \cdot s)$ & -50 & 250 \\
\hline LSH-S 350 & 7 & $f \cdot(7-8 \cdot s)$ & -50 & 350 \\
\hline
\end{tabular}

Table III. PSH components according to (11) in $\mathrm{Hz}$

\begin{tabular}{|c|r|r|c|c|}
\hline & $\boldsymbol{\lambda}$ & $\boldsymbol{v}$ & $f_{R S H}(s=1)$ & $f_{R S H}(s=0)$ \\
\hline PSH-5 & 1 & -5 & -250 & 450 \\
\hline PSH-3 & 1 & -3 & -150 & 550 \\
\hline PSH-1 & 1 & -1 & -50 & 650 \\
\hline PSH+1 & 1 & 1 & 50 & 750 \\
\hline PSH+3 & 1 & 3 & 150 & 850 \\
\hline
\end{tabular}

Table IV. Eccentricity components according to (13) in $\mathrm{Hz}$

\begin{tabular}{|c|c|c|c|}
\hline Component & $\boldsymbol{m}$ & $\begin{array}{c}\text { Initial frequency } \\
\text { value (connection, } \\
s=1)\end{array}$ & $\begin{array}{c}\text { Final frequency } \\
\text { value (steady- } \\
\text { state, } s=0)\end{array}$ \\
\hline EC 25 & -1 & 50 & 25 \\
\hline EC 75 & 1 & 50 & 75 \\
\hline EC 100 & 2 & 50 & 100 \\
\hline EC 125 & 3 & 50 & 125 \\
\hline
\end{tabular}

